\title{
Interfaces
}

\section{Le bestiaire d'Alexis Fraikin à la Galerie Laurent de Puybaudet}

\section{Gabrielle Thierry}

\section{(2) OpenEdition}

1 Journals

Édition électronique

URL : http://journals.openedition.org/interfaces/1932

DOI : 10.4000/interfaces. 1932

ISSN : 2647-6754

Éditeur :

Université de Bourgogne, Université de Paris, College of the Holy Cross

\section{Édition imprimée}

Date de publication : 15 décembre 2020

Pagination : 179-190

ISSN : 1164-6225

\section{Référence électronique}

Gabrielle Thierry, « Le bestiaire d'Alexis Fraikin à la Galerie Laurent de Puybaudet », Interfaces [En ligne], 44 | 2020, mis en ligne le 15 décembre 2020, consulté le 21 décembre 2020. URL : http:// journals.openedition.org/interfaces/1932 ; DOI : https://doi.org/10.4000/interfaces.1932

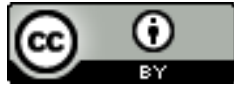

Les contenus de la revue Interfaces sont mis à disposition selon les termes de la Licence Creative Commons Attribution 4.0 International. 


\title{
LE BESTIAIRE D'ALEXIS FRAIKIN À LA GALERIE LAURENT DE PUYBAUDET
}

\author{
Gabrielle Thierry \\ «Tout parle en mon ouvrage, même les poissons : ce qu'ils disent \\ s'adresse à tous tant que nous sommes. Je me sers d'animaux pour \\ instruire les hommes ", Jean de La Fontaine ${ }^{1}$
}

\begin{abstract}
À Paris, dans le cour historique, la galerie Laurent de Puybaudet a une place singulière, elle est située à un carrefour fort étroit et mérite par son apparence le nom de proue. Cette proue est celle du bateau qui transporte les animaux rares et en voie d'extinction de l'artiste Alexis Fraikin. Il avance dans cette rue à évocation fluviale, la rue de Seine. C'est ici que je rencontre Laurent de Puybaudet et Alexis Fraikin pour une immersion dans ce monde sillonné de bêtes colorées. Je réaliserai vite qu'il s'agit là pour l'artiste de faire bouger les lignes et de nous révéler - à pas feutrés - la tyrannie des hommes sur le monde animal, notre environnement, nos écosystèmes.
\end{abstract}

\section{La Galerie}

Laurent de Puybaudet a ouvert cette galerie par passion il y a 20 ans. Il y expose et défend les artistes $\mathrm{du} \mathrm{XX}^{\mathrm{e}}$ siècle dont l'art singulier, l'art brut et l'art populaire s'expriment sous toutes leurs formes : bois, fer, céramique et particulièrement les œuvres sur papier, poétiques et rêveuses.

Les trois vitrines qui composent la coque de cette arche de Noé, présentent dans de petits cadres bruns les dessins travaillés d'Alexis Fraikin. Je découvre pour la première fois les œuvres de l'artiste, en laissant vivre en moi l'émotion colorée et graphique. Une tortue, une poule, une hirondelle, un lièvre, une grenouille, se côtoient sous le chêne et le roseau. Certaines de ces images m'évoquent immédiatement quelques vers de La Fontaine.

Issue de la dédicace adressée à Louis de France, fils du roi Louis XIV. Le Dauphin est âgé de sept ans lorsque La Fontaine lui dédie son recueil de fables. 


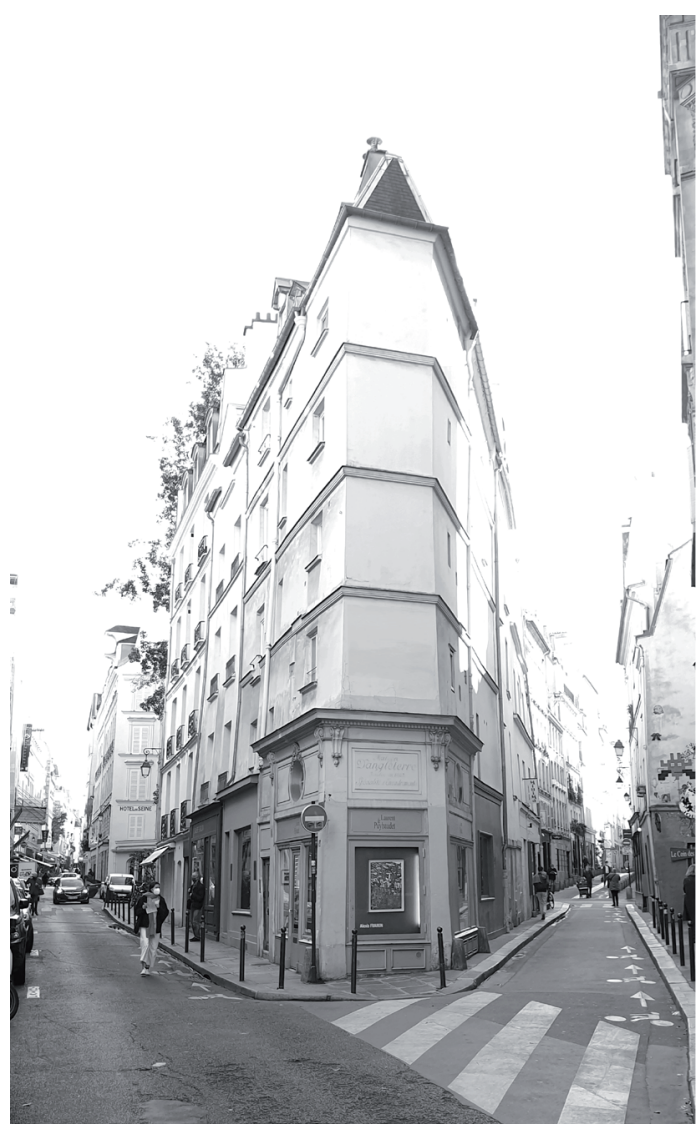

Figure 1. Galerie Laurent de Puybaudet. (c) Gabrielle Thierry, 2020.

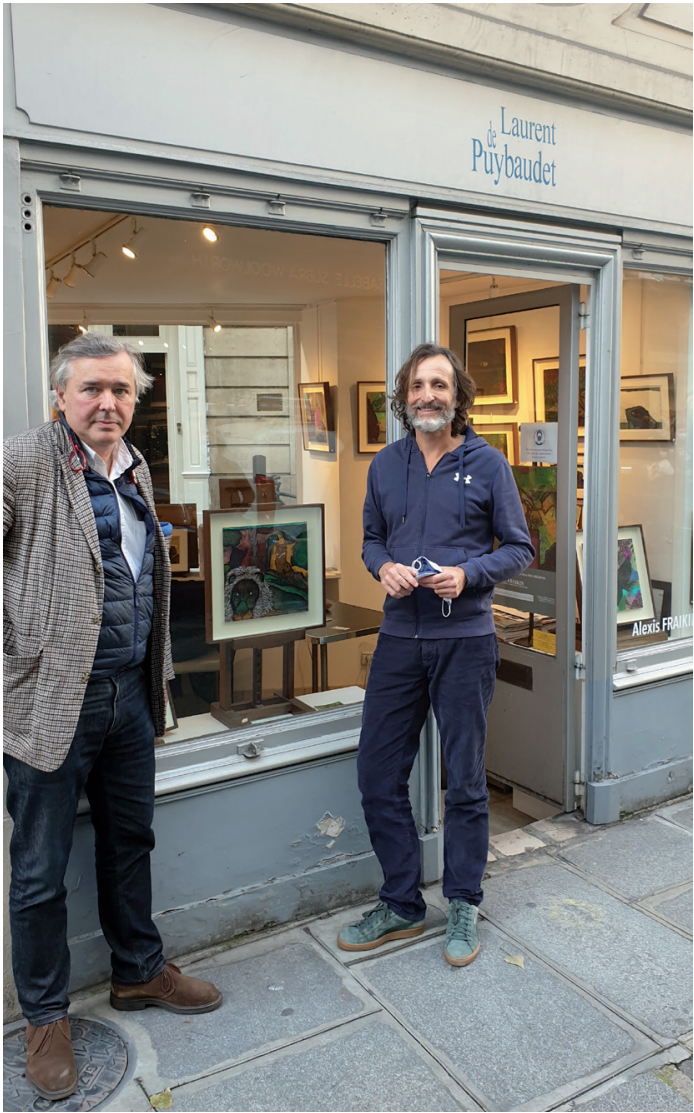

Figure 2. Laurent de Puybaudet et Alexis Fraikin. (C) Gabrielle Thierry, 2020.

\section{L'artiste}

Alexis Fraikin est un autodidacte et peint la nature entre figuration et abstraction. Il privilégie le dessin depuis dix ans. Son support est le papier, le stylo, l'encre et l'aquarelle. L'exposition présente une série de dessins originaux destinés à l'édition de l'ouvrage La Fontaine et l'étang moderne, titre jouant sur 
les mots des « temps modernes ». De très courts textes, écrits par la journaliste Elsa Launay, dénoncent le drame des disparitions silencieuses des espèces et l'effondrement en cours du monde du vivant. Je parcours le livre.

\section{L'exposition-livre}

Les dessins originaux exposés au mur dans leur cadre sont soudainement indissociables du livre. L'ouvrage au format carré $(21 \times 21 \mathrm{~cm})$ a été édité par la maison d'édition le Ravin Bleu en 2019. Chaque double page est composée du texte à gauche et de son illustration à droite, soit 30 doubles pages pour 30 dessins.

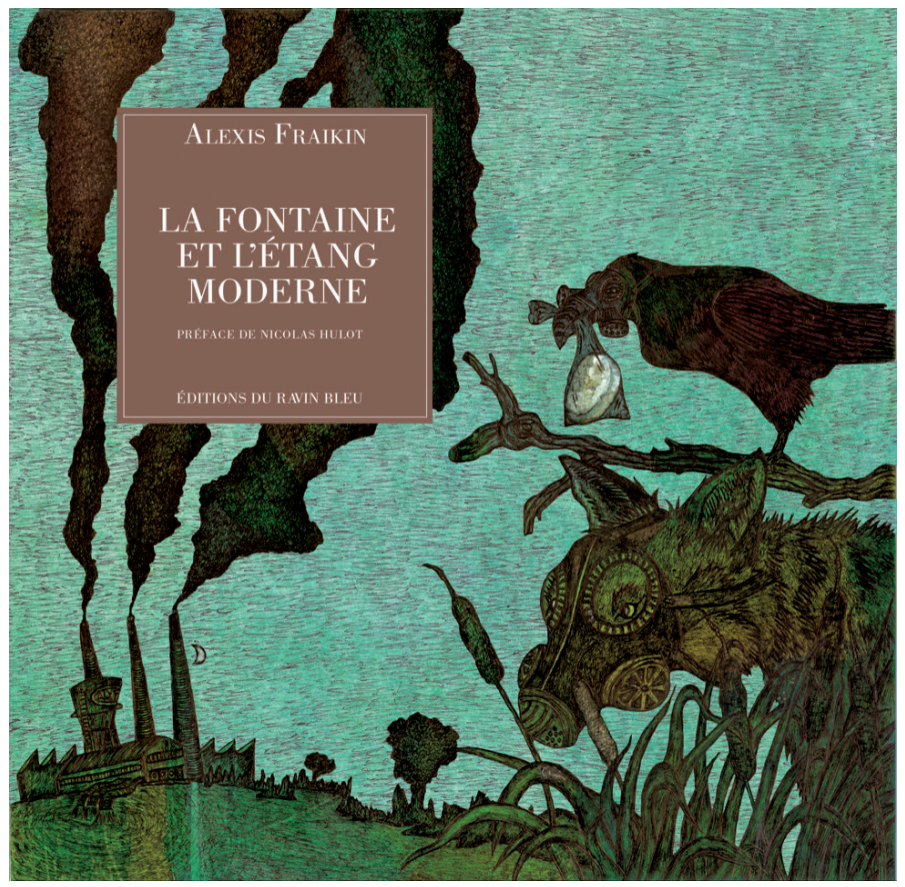

Figure 3. La Fontaine et l'étang moderne A.Fraikin et E.Launay, Ed. RavinBleu, 2019.

(c) Alexis Fraikin 2020. 

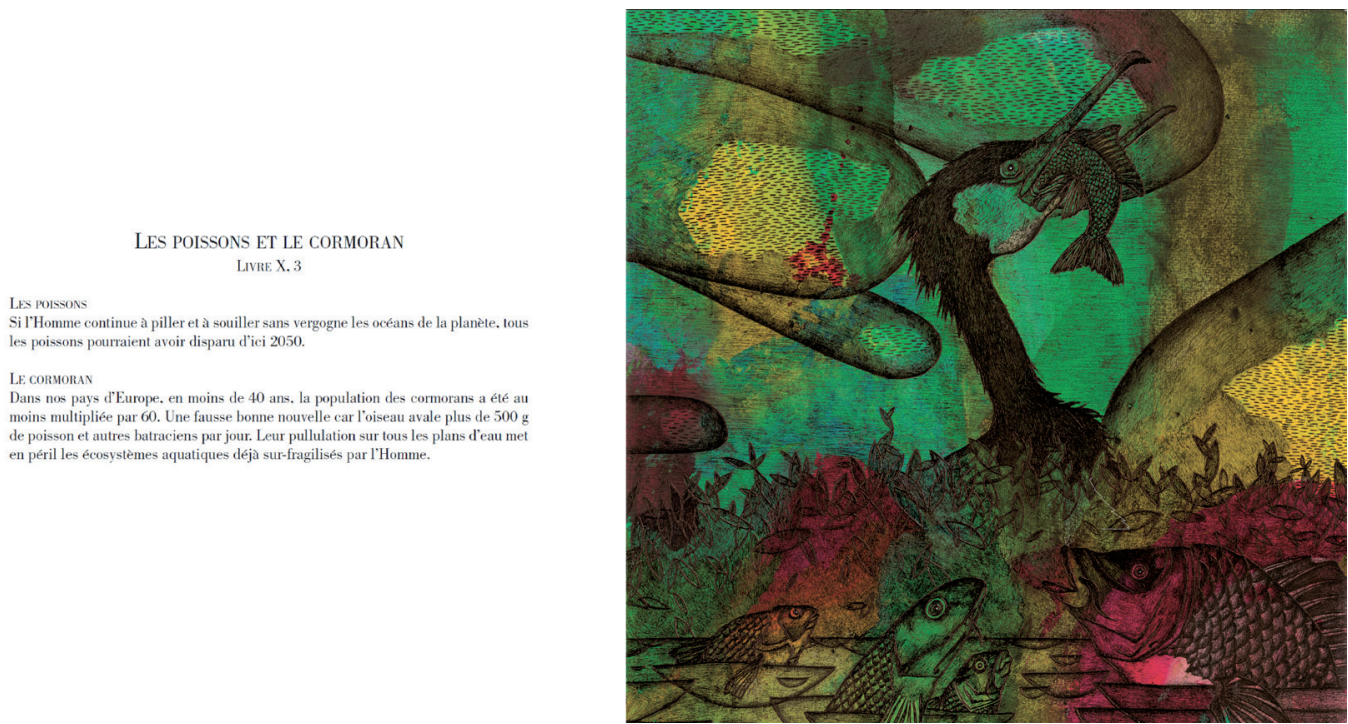

Figure 4. «Les poissons et le cormoran », double page de l'ouvrage La Fontaine et l'étang moderne.

(C) Alexis Fraikin 2020.

A gauche, le titre de la Fable de la Fontaine est accompagné de sa référence au livre original. Suit le texte critique remplaçant celui de la fable de la Fontaine, à l'origine du choix iconographique de l'artiste. Ici, pas de vers, pas de rime, pas de poésie, mais les chiffres crus d'un constat écologique de l'état actuel de notre environnement.

«Le cheval et l'âne » permet de faire constat des tueries de chevaux en France et du massacre des ânes au Kenya pour leur peau et leurs vertus médicinales.

Dans « Le chêne et le roseau », l'artiste veut dénoncer les déforestations.

Tandis que dans « Les poissons et le cormoran » l'artiste nous rappelle en quelques chiffres l'état de nos océans pollués ; les poissons pourraient avoir disparu d'ici 2050. La multiplication excessive des cormorans est une fausse bonne nouvelle; du fait qu'ils sont très gourmands en êtres vivants aquatiques, leur surpopulation met en péril tout un écosystème.

À droite, l'illustration est imprimée sur toute la surface de la page, sans marge. 


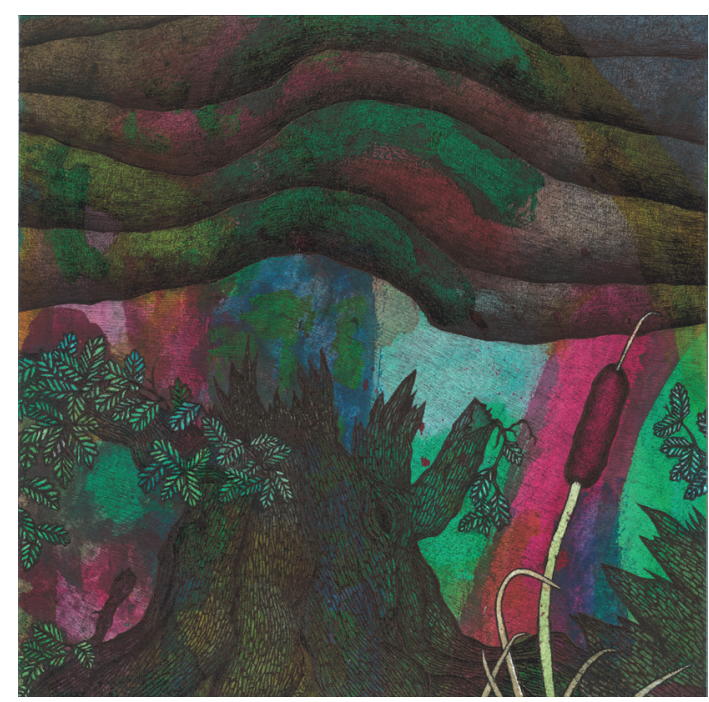

Figure 5. « Le chêne et le roseau », 30x30cm, stylobille et encres sur papier, 2019.

(C) Alexis Fraikin 2020.

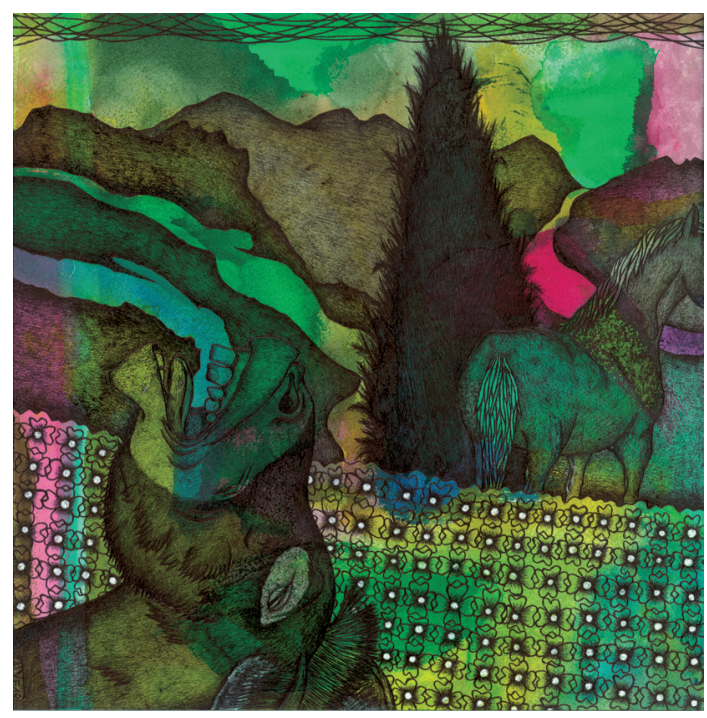

Figure 6. « Le cheval et l'âne », 30x30cm, stylo-bille et encres sur papier, 2019.

(C) Alexis Fraikin 2020.

En ouvrant le livre, en lisant le texte, je décrypte différemment le dessin en regard.

Alexis Fraikin se sert du bestiaire de La Fontaine comme point de départ graphique. Il s'intéresse à la mise en situation des animaux du poète pour dessiner son propos et lancer ses alertes. Les fables sont donc sélectionnées en fonction des espèces mentionnées et en privilégiant celles dont l'extinction est imminente.

L'artiste, très concerné par ce sujet, rappelle la situation dramatique de notre écosystème. Il veut éviter de reprendre les discours " classiques » sur la sixième extinction de masse à venir et le réchauffement climatique. Son expression se veut plus poétique. 


\section{L'œuvre}

Au premier coup d'œil, se révèlent les dessins enchanteurs, gravés dans des atmosphères aux couleurs sombres, majorées de verts des sous-bois. Je m'attends à voir ou lire les fables connues de tous alors que les titres agissent en trompe l'œil.

Découpé au format toujours carré pour l'harmonie, le format parfait selon l'artiste, le papier est peint à l'encre aquarelle. Dans cette première étape, la surface est recouverte aléatoirement de couleurs : les bleus-verts apparaissent intuitivement, quelques rouges rosés illuminent ces fonds ondoyants et vaporeux. Après le séchage, les couleurs sont parfois retravaillées, ajoutant de la profondeur à ce décor sombre. Sur ces espaces, l'artiste vient dessiner ses scènes au stylo. Le dessin est ample, les formes sûres et le stylo passe et repasse dans son premier sillon. Après plusieurs passages, le noir de l'encre prend une apparence cuivrée et le dessin ressemble à une gravure. Les couleurs sont grattées, maltraitant par endroit le papier épais, sec et grammé. Ces nouveaux espaces blancs et feutrés sont retouchés pour atténuer les contrastes. Des formes sans signification particulière renforcent l'équilibre de la composition.

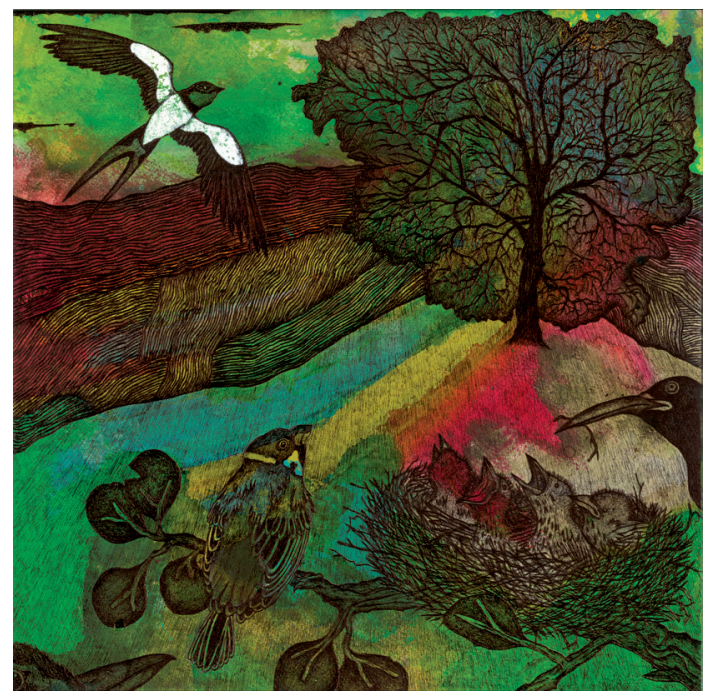

Figure 7. «L'hirondelle et les petits oiseaux », 30x30cm, stylo-bille et encres sur papier, 2019.

(C) Alexis Fraikin 2020. 
Le temps n'est pas compté dans la réalisation de ces œuvres de petits formats (entre 20 et 40 $\mathrm{cm}$ ). Le dessin occupe tout l'espace de la feuille, aucune marge n'est présente. Le dessinateur étend son motif jusqu'aux limites de la feuille.

Le dessin est figuratif, les animaux, sans aucune transformation, sont reconnaissables. Il n'y a pas d'allégorie, de dieu, ni de monstre, qui appartiennent plutôt au monde de l'art brut.

Pour la réalisation du dessin de «L'hirondelle et les petits oiseaux », lorsque l'artiste perçoit un champ dans l'impression des mouvements des fonds encrés, il décide d'y faire voler l'hirondelle, ses ailes sont blanchies par grattage. Il trame le champ pour lui donner du corps. Au tout premier plan sont positionnés sur une branche les oiseaux et leur nid. Dans l'espace libre, au-dessus, se dessine un arbre de dentelle.

Sur ce paysage au fond émeraude et prune, les oiseaux chantent encore. Le texte associé rappelle un constat accablant : « En 17 ans, 1/3 des oiseaux a disparu des campagnes françaises. Les causes principales de ce déclin sont l'agriculture intensive et l'utilisation massive des pesticides. »

\section{Les fables, l'illustration et l'emprunt}

L'illustration des fables se pratique avec l'enluminure et les figures d'animaux sous forme satirique envahissent les marges des manuscrits dès le XIII' siècle. L'exposition virtuelle sur le bestiaire médiéval sur le site de la BnF est éclairant sur ce point ${ }^{2}$. 


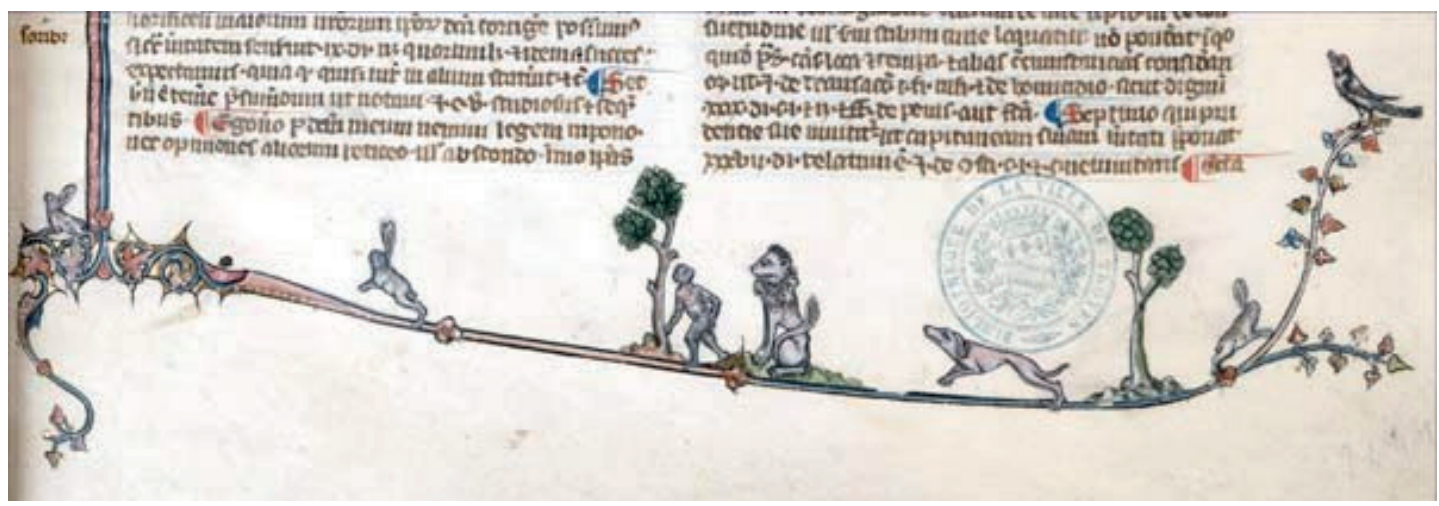

Figure 8. La figure des animaux dans les marges des manuscrits, cette pratique disparaît avec l'imprimerie.

Site de l'exposition sur le bestiaire au moyen âge http://expositions.bnf.fr/bestiaire/arret/1/index.htm

En 1668, la première édition des fables de La Fontaine est illustrée de petites gravures de François Chauveau, graveur célèbre de son époque ${ }^{3}$.

Grâce à l'illustration, le lecteur « voit » la fable avant de la lire. Il interprète l'image différemment après la lecture. Le texte peut aussi être compris, interprété d'une autre manière en fonction de l'image. Cette interface enrichit le dialogue entre texte et image.

3. Au fil des siècles, d'autres illustrateurs s'attaqueront à la mise en image des fables, citons les plus ambitieux : 275 dessins du peintre de natures mortes et de scènes de chasse Baptiste Oudry au XVIII ${ }^{\mathrm{e}}$ siècle ; le caricaturiste Granville, dont la satire excessive sera à la l'origine de la loi exigeant une autorisation préalable pour toute publication; Gustave Doré multipliant les points de vue graphiques avec une recherche d'efficacité sur l'image, au XIX ${ }^{\mathrm{e}}$ siècle. En 1905, c'est au tour de Benjamin Rabier, et Calvet Rognat dont les illustrations sont entrées dans l'imagerie populaire tant elles ont envahi les manuels scolaires. 

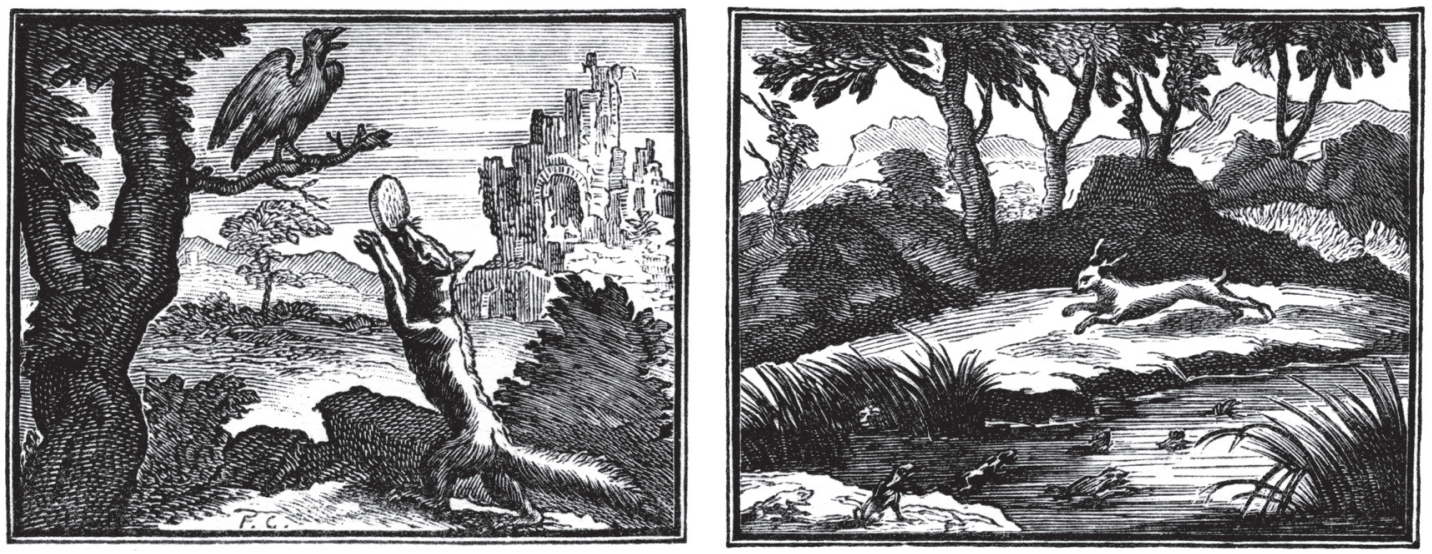

Figure 9. Gravures de François Chauveau, édition des Fables de La Fontaine, 1668. https://commons.wikimedia.org/wiki/Category:Illustrations of Jean_de La_Fontaine $\% 27 \mathrm{~s}$ fables by Fran\%C3\%A7ois_Chauveau

Aujourd'hui il est dommage que certaines éditions contemporaines des fables de La Fontaine ne comprennent pas d'illustration.

Dans sa poésie, le fabuliste crée des dialogues entre les êtres pour explorer les méandres de la nature humaine. Il y a de la gaieté, de l'humour et parfois des drames. Les Fables divertissent. Les protagonistes s'entendent rarement. Les désirs des hommes provoquent des tensions. Les bêtes se jugent, se jaugent, se moquent.

Si Alexis Fraikin emprunte à Jean de La Fontaine ses titres, son bestiaire, il ne fait pas parler les poissons, pas plus qu'il n'humanise la faune et la flore. L'animal reste animal et représente son espèce. En guise de moralité, les deux auteurs nous révèlent les constats écologiques publiés sur les autres médias.

La Fable est un genre qui se transforme au gré des époques. Jean de La Fontaine les a empruntées au poète Esope. 11 se les ait appropriées tout en les détournant pour mieux nous interpeller et nous divertir. Il les a modernisées grâce à une immense connaissance de l'Antiquité et de la Renaissance. Il a mis en scène de nouveaux animaux pour aller plus loin dans l'exploration de l'âme humaine. 


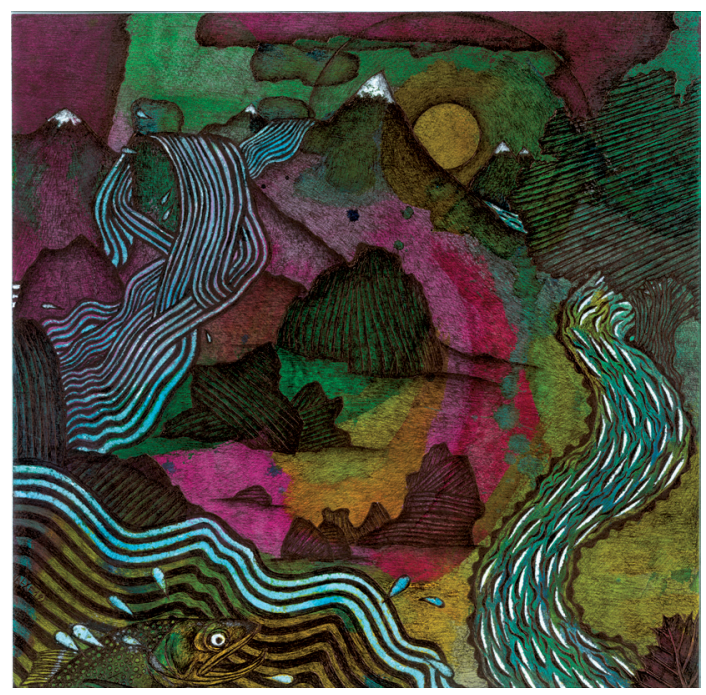

Figure 10. « Le torrent et la rivière », 30x30cm, stylo-bille et encres sur papier, 2019.

(C) Alexis Fraikin 2020.

A. Fraikin emprunte les animaux de La Fontaine. Il les déshabille de toute humanité, leur retire la voix, les intentions. Les voilà muets, parés uniquement de leurs atours d'animaux.

\section{L'intention}

Dans le processus de création de ces images, de cet ouvrage, il y a une attention bien particulière et les visiteurs de la galerie ne réagissent pas toujours de la même manière. Certains expriment leur déception quant à l'éloignement du texte initial du fabuliste. D'autres, au contraire, se sentent concernés par la démarche écologique et politique, et apprécient l'emprunt et le détournement opérés par l'artiste. 


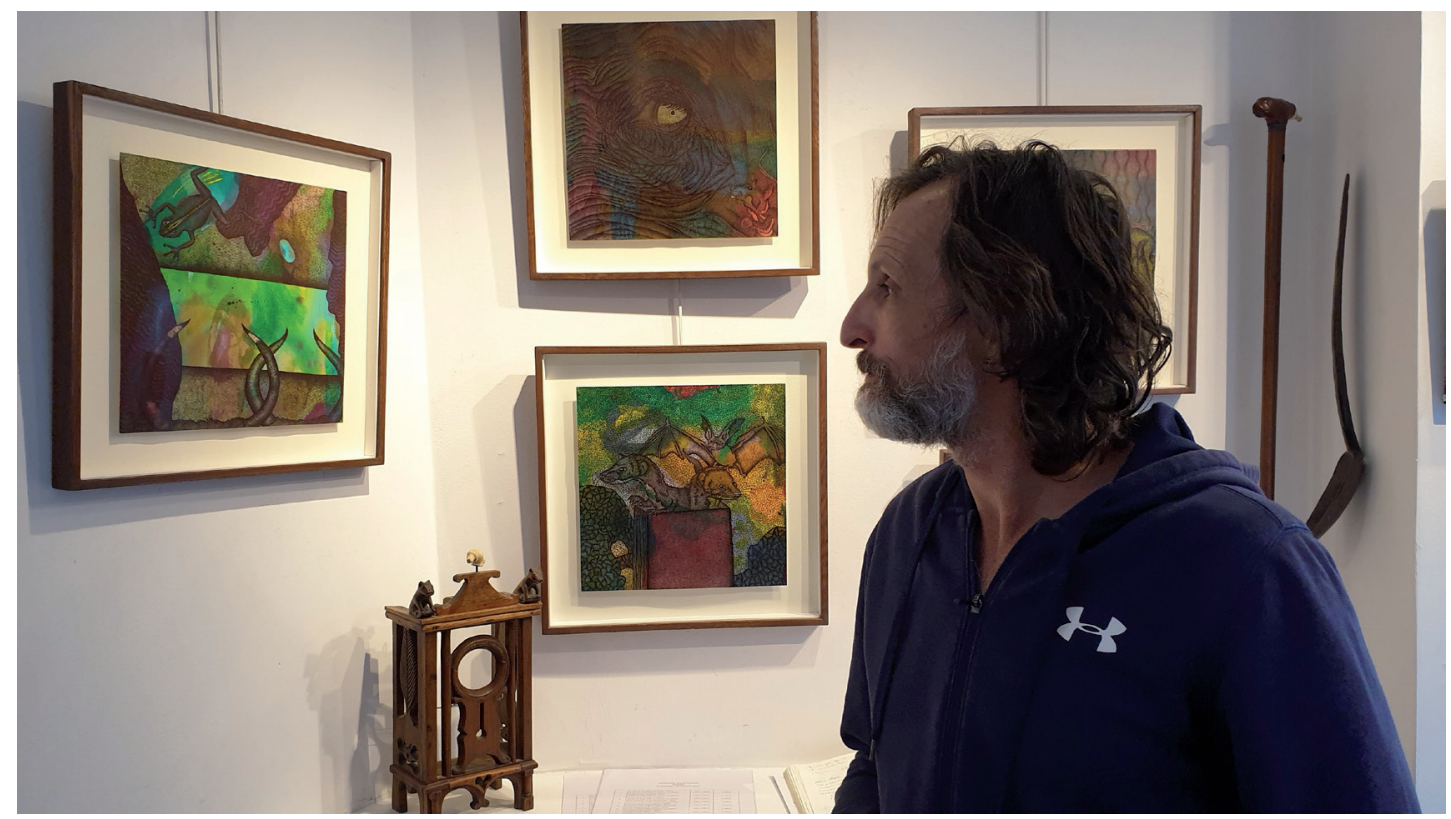

Figure 11. Alexis Fraikin devant ses œuvres.

(C) Gabrielle Thierry, 2020.

Le stylo - non pour écrire mais pour décrire, dessiner - enchante étrangement ces images muettes. Les illustrations racontent une autre histoire que celle des fables, celle de ces animaux bien réels, devenus vulnérables dans un environnement de plus en plus hostile.

Alexis Fraikin fait partie de ces nouveaux artistes adhérant à l'écologie. ÀA la protestation, il préfère la poétique du dessin, l'attrait des fables et une représentation de la faune et de la flore gravées dans un monde au décor sombre. Les Fables de la Fontaine sont donc un prétexte à l'expression artistique d'une angoisse, celle de l'artiste face à la perspective d'un désastre écologique. 


\section{RÉFÉRENCES}

BESTIAIRE DU MOYEN ÂGE, exposition à la Bibliothèque Nationale de France: URL : www.expositions. bnf.fr/bestiaire (page consultée le 18 novembre 2011).

DANDREY, Patrick. La Fontaine ou les métamorphose d'Orphée (Gallimard Découvertes 1995, 2008), à écouter dans l'émission intitulée " Le paradis perdu de Jean de La Fontaine » lors des Entretiens de Matthieu Garrigou-Lagrange dans La compagnie des œuvres sur France Culture. https://www.franceculture.fr/ emissions/la-compagnie-des-auteurs/jean-de-la-fontaine-14-le-paradis-perdu-de-jean-de-la-fontaine (page consultée le 18 novembre 2020)

LEPLÂTRE, Olivier. La Parole et le pouvoir dans les Fables de La Fontaine (PU de Lyon 2002), à écouter dans l'émission «La pensée et la sagesse selon La Fontaine » lors des Entretiens de Matthieu GarrigouLagrange dans La compagnie des ouvres sur France Culture. https://www.franceculture.fr/emissions/ la-compagnie-des-auteurs/jean-de-la-fontaine-34-la-pensee-et-la-sagesse-selon-la-fontaine (page consultée le 18 novembre 2020)

SITE DE LA GALERIE LAURENT DE PUYBAUDET, www.galeriedepuybaudetblog.wordpress.com 